\title{
Graphene quantum dots disrupt embryonic stem cell differentiation through interfering methylation level of Sox2
}

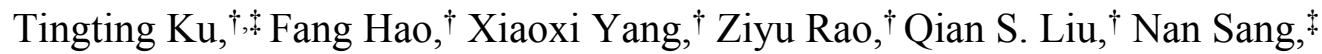
Francesco Faiola, ${ }^{\dagger}, \S$ Qunfang Zhou, ${ }^{*}, \dagger, \S, \|$ Guibin Jiang ${ }^{\dagger}, \S, \|$

†State Key Laboratory of Environmental Chemistry and Ecotoxicology, Research Center for Eco-Environmental Sciences, Chinese Academy of Sciences, Beijing 100085, China

tCollege of Environment and Resource, Research Center of Environment and Health, Shanxi University, Taiyuan, 030006, China

$\S$ College of Resources and Environment, University of Chinese Academy of Sciences, Beijing 100049, China

ISchool of Environment, Hangzhou Institute for Advanced Study, University of Chinese Academy of Sciences, Hangzhou, 310000, China

\footnotetext{
* Correspondence to:
}

Dr. Qunfang Zhou, State Key Laboratory of Environmental Chemistry and Ecotoxicology, Research Center for Eco-Environmental Sciences, Chinese Academy of Sciences, Beijing 100085, China.

E-mail: zhouqf@,rcees.ac.cn 
Contents:

29

Figure S1-S4

30

Table S1

31

32

Supplementary Figures

33

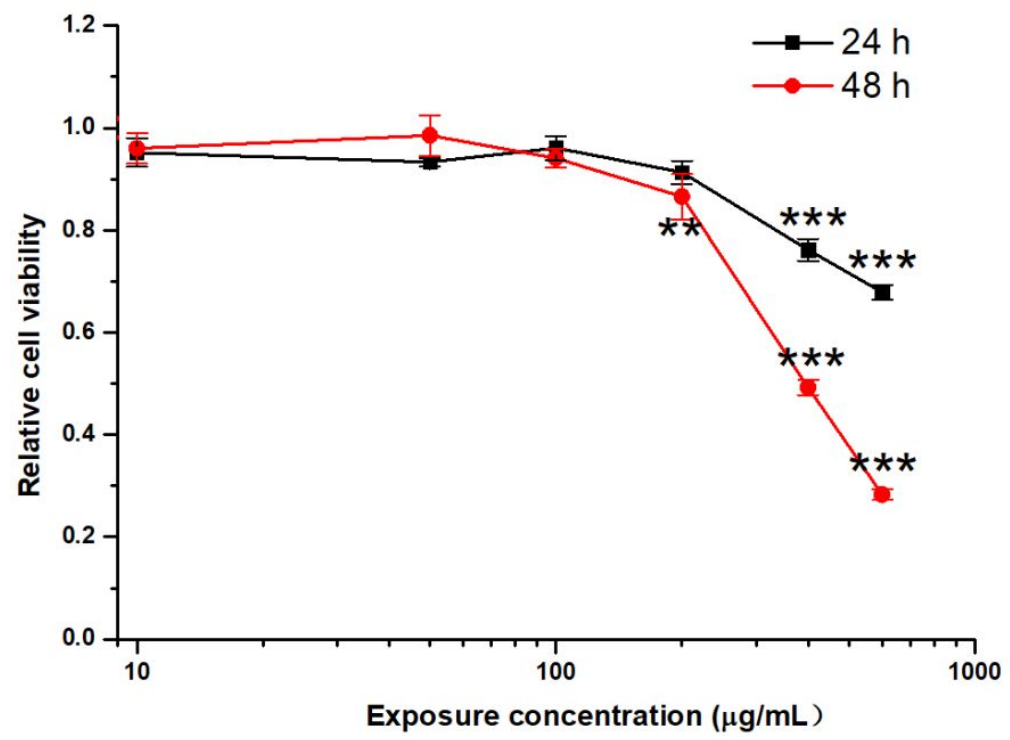

34

Figure S1. The cell viabilities of the $\operatorname{mESCs}(n=6) .{ }^{* *} p<0.01$, and ${ }^{* * *} p<0.001$ versus control. 


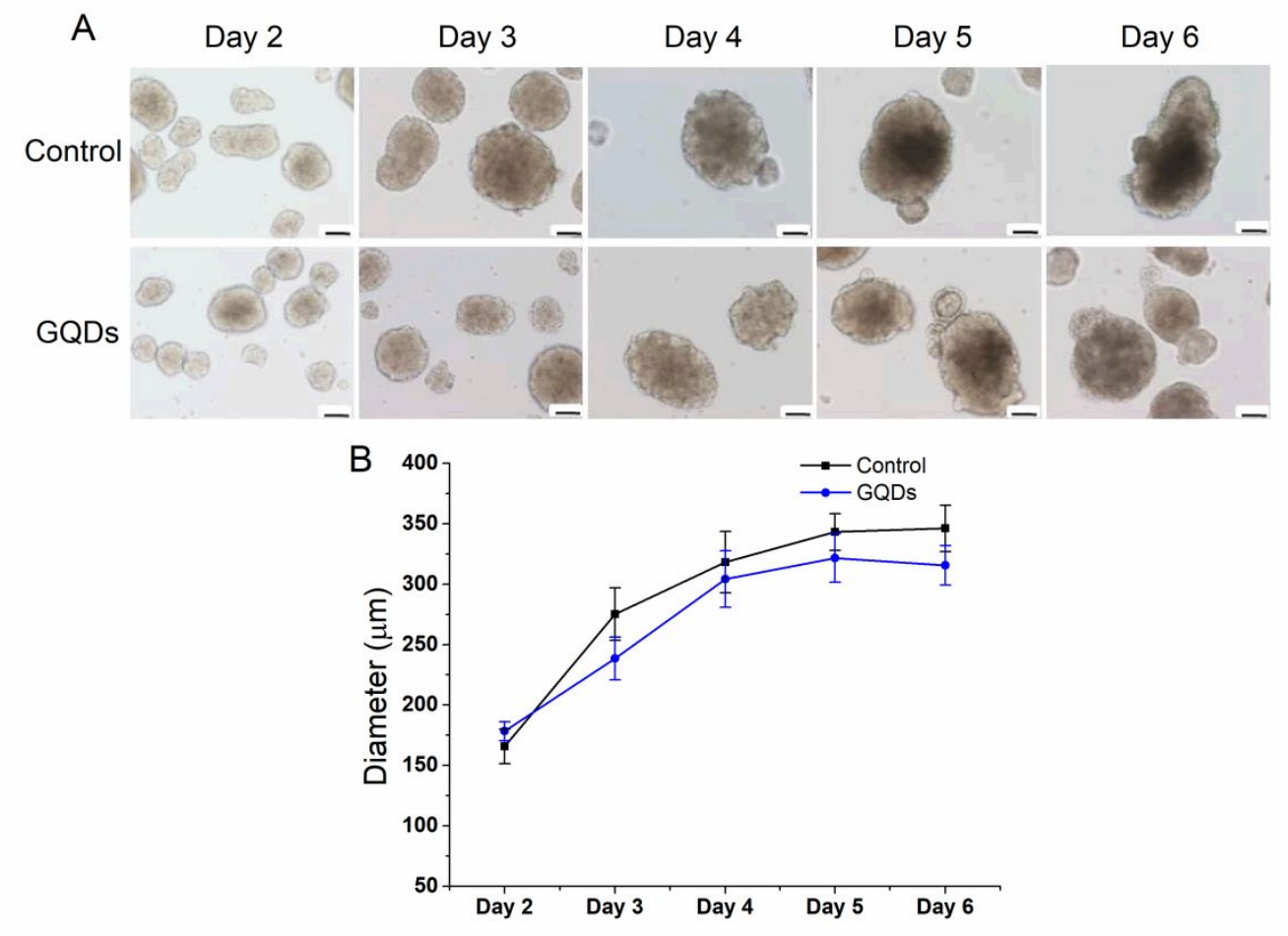

Figure S2. Characterization of EBs. (A) Morphological observation of the 39 developing EBs. Scale bar $=100 \mu \mathrm{m}$. (B) The colony sizes of EBs with or without 40 GQD pretreatment $(50 \mu \mathrm{g} / \mathrm{mL}, 24 \mathrm{~h})$. 
A

$$
\begin{aligned}
& \text { EB }
\end{aligned}
$$

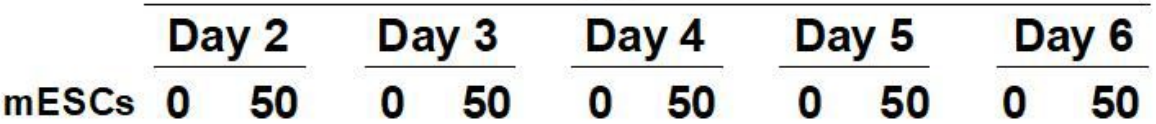

$$
\begin{aligned}
& \text { mESCs } 0 \quad 50 \text { } 0 \text { 50 }
\end{aligned}
$$

\section{Brachyury}

\section{Krt14}

GAPDH

\section{B}
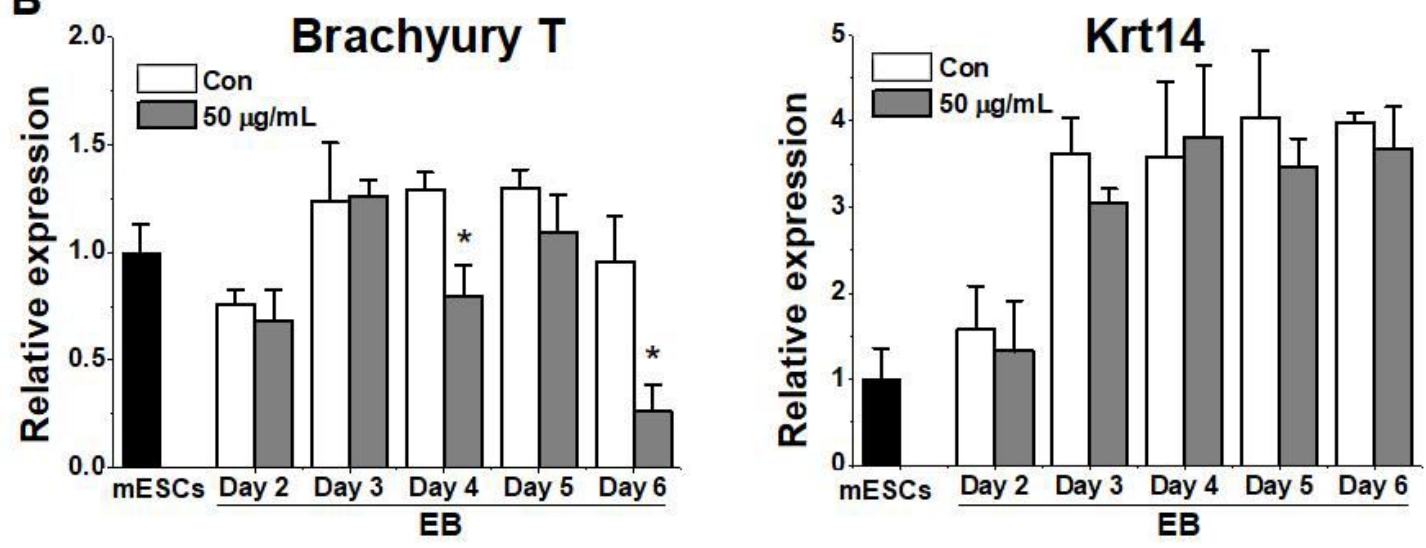

Figure S3. The influence of GQDs on differentiation biomarkers $(n=3)$. (A) Western blotting for the expressions of Brachyury $\mathrm{T}$ (mesoderm) and Krt14 (ectoderm) in EBs collected on different days. (B) Quantitative analysis for the protein levels of the test biomarkers $(n=3) .{ }^{*} p<0.05$ versus the control at corresponding time points. 


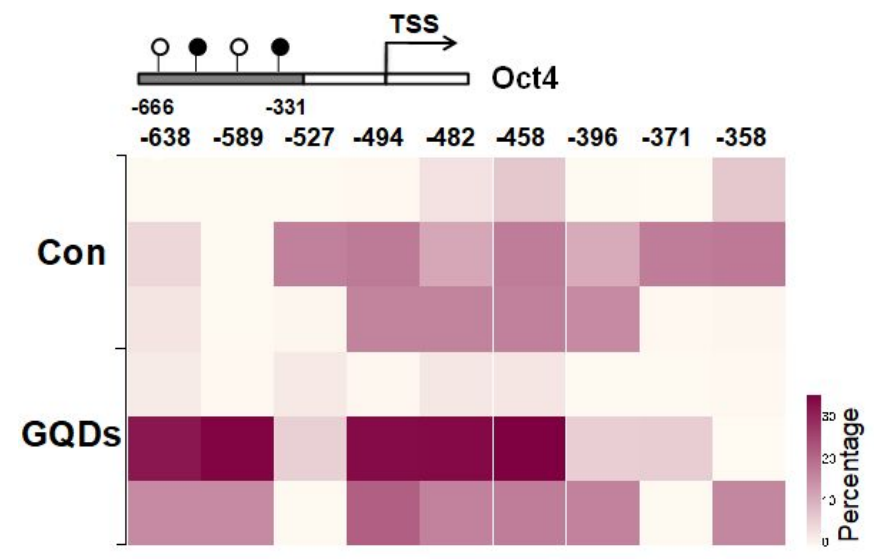

47

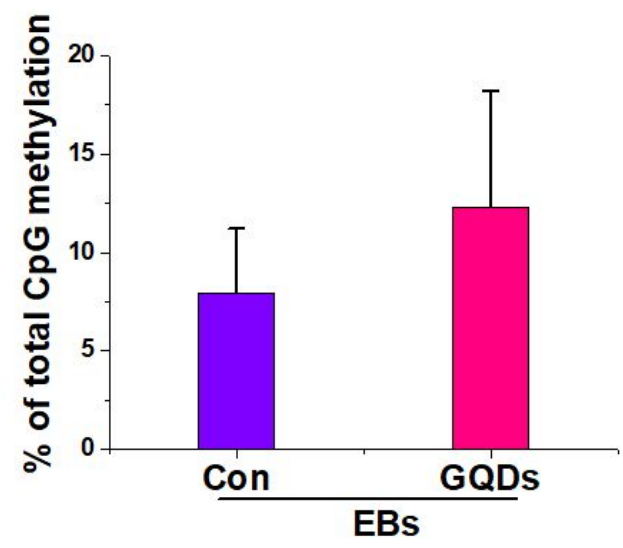

48 Figure S4. DNA methylation status of Oct4 promoter region in EBs $(n=3)$. TSS:

49 Transcription start sites. The exposure concentration of GQDs was $50 \mu \mathrm{g} / \mathrm{mL}$, and the 50 stimulation duration was $24 \mathrm{~h}$. 


\section{Supplementary Table}

53 Table S1. The primer sequences for $q$ PCR analysis.

\begin{tabular}{|c|c|c|}
\hline \multirow{2}{*}{ Gene name } & \multicolumn{2}{|c|}{ Sequence of the primers $\left(5^{\prime}-3^{\prime}\right)$} \\
\hline & Forward & Reverse \\
\hline GAPDH & CCTGCGACTTCAACAGCAAC & TAGGGCCTCTCTTGCTCAGT \\
\hline Sox 2 & AACCGATGCACCGCTACGA & TGCTGCGAGTAGGACATGCTG \\
\hline Oct 4 & TGGATCCTCGAACCTGGCTA & CTCAGGCTGCAAAGTCTCCA \\
\hline Nanog & GGAGGACTTTCTGCAGCCTT & TGCCCTGACTTTAAGCCCAG \\
\hline Sox17 & GATGCGGGATACGCCAGTG & CCACCACCTCGCCTTTCAC \\
\hline GATA6 & TACACAAGCGACCACCTCAG & TGTAGAGGCCGTCTTGACCT \\
\hline Mesp1 & CAGAATCGTGGGACCCATCGTT & TGAAGAGCGGAGATGAGGGACT \\
\hline Brachyury $T$ & GTCTAGCCTCGGAGTGCCT & CCATTGCTCACAGACCAGAG \\
\hline Krt14 & GGCCCACTGAGATCAAAGAC & GATCTGCAGGAGGACATTGG \\
\hline$F g f 5$ & GCTGTGTCTCAGGGGATTGT & ACAGTCATCCGTAAATTTGGC \\
\hline Dnmt1 & ACCATCACGGCTCACTTCAC & GGGTTCCCGCTGTTACCTCTT \\
\hline Dnmt3a & CCAAGAAACCCAGAAAGAGCA & ATCGTCGTCATACTGGTAAGCACA \\
\hline$D n m t 3 b$ & CACCAACAACAAGGGCAATC & AGCACCTCCAGACACTCCAC \\
\hline Tet 1 & GAGCCTGTTCCTCGATGTGG & CAAACCCACCTGAGGCTGTT \\
\hline Tet2 & AACCTGGCTACTGTCATTGCTCCA & ATGTTCTGCTGGTCTCTGTGGGAA \\
\hline Tet 3 & TCCGGATTGAGAAGGTCATC & CCAGGCCAGGATCAAGATAA \\
\hline
\end{tabular}

54 